\title{
Warning Symptoms and Family History in Children and Young Adults with Sudden Cardiac Arrest
}

\author{
Jonathan A. Drezner, MD, Jessie Fudge, MD, Kimberly G. Harmon, MD, \\ Stuart Berger, MD, Robert M. Campbell, MD, and Victoria L. Vetter, MD
}

Background: Children and young adults with undiagnosed cardiovascular disorders at risk for sudden death may have warning symptoms or significant family history that is detectable through screening. The objective of this study was to determine the prevalence of warning symptoms and family history in a cohort of children and young adults who suffered sudden cardiac arrest (SCA).

Methods: A retrospective survey investigating warning symptoms and family history of cardiovascular disease was completed by families with a child or young adult who suffered SCA.

Results: Eighty-seven of 146 families (60\%) returned a completed survey. The SCA victims were an average age of 16 years (range, <5-29 years), 69\% male, and 68\% white. Seventy-two percent of SCA victims were reported by their parents to have at least one cardiovascular symptom before SCA, with fatigue (44\%) and near-syncope/lightheadedness (30\%) the two most common. Twenty-four percent of SCA victims had one or more (average 2.6; range, 1 to 10) events of syncope or unexplained seizure that remained undiagnosed as a cardiac disorder before SCA. Parents reported that cardiovascular symptoms first occurred, on average, 30 months (range, 19 to 71 months) before SCA; a symptom was brought to the attention of the child's physician in $41 \%$ of cases. Twenty-seven percent of families reported a family member had suffered sudden death before age 50 because of a heart condition.

Conclusions: Many children and young adults who suffered SCA are reported to have cardiac symptoms or a family history of premature cardiac death. Syncope and unexplained seizure activity are distinct events but often go unrecognized as ominous signs of underlying cardiovascular disease. Physician education and increased public awareness regarding cardiovascular warning signs in the young may improve early detection of those at risk and prevent tragedies. (J Am Board Fam Med 2012;25: 408-415.)

Keywords: Cardiovascular Abnormalities, Prevention, Retrospective Study, Screening, Sports Medicine, Sudden Cardiac Death

Sudden cardiac arrest (SCA) is the leading cause of nontraumatic sudden death in children and young adults and usually is the result of an undiagnosed cardiovascular disorder. ${ }^{1,2}$ The causes of pediatric and young adult SCA involve a heterogeneous

This article was externally peer reviewed.

Submitted 20 July 2011; revised 23 December 2011; accepted 12 January 2012.

From the Department of Family Medicine, University of Washington, Seattle (JAD, JF, KGH); the Department of Pediatric Cardiology, Children's Hospital of Wisconsin, Milwaukee (SB); the Department of Pediatric Cardiology, Sibley Heart Center, Children's Healthcare of Atlanta, Atlanta, GA (RMC); the Department of Pediatric Cardiology, Children's Hospital of Philadelphia, Philadelphia, PA (VLV).

Funding: none.

Conflict of interest: none declared. group of cardiac diseases including structural cardiovascular abnormalities, such as cardiomyopathies, and primary cardiac electrical diseases, such as ion channel disorders. The prevalence of warning signs or symptoms in children and young adults who later suffer SCA is highly variable and poses a challenge to identifying through screening persons at elevated risk of sudden cardiac death (SCD). ${ }^{3}$ Sudden death is the first clinical manifestation of underlying cardiovascular disease in up to $50 \%$ to $80 \%$ of young athletes with SCD. ${ }^{4-7}$

Corresponding author: Jonathan A. Drezner, MD, Department of Family Medicine, University of Washington, Box 354410, Seattle, WA 98195 (E-mail: jdrezner@fammed. washington.edu). 
Not much is known about the potential to identify children and young adults at elevated risk of SCD through a comprehensive symptom and family history. During routine well-child evaluations, little attention may be given to a focused cardiovascular risk assessment. In young athletes, preparticipation cardiovascular screening is routinely recommended by most major sporting and medical associations including the American Heart Association (AHA). ${ }^{8-11}$ However, universal endorsement of a single screening strategy to identify athletes at risk for SCD remains elusive and a topic of tremendous debate within the sports medicine and cardiology communities. In addition, recommendations for use of a comprehensive medical and family history questionnaire to guide the preparticipation evaluation have not been widely adopted in practice, and screening protocols often are implemented inadequately. ${ }^{12-14}$ The management and subsequent evaluation of children with warning symptoms also may be incomplete, with the potential to overlook underlying cardiovascular pathology. The purpose of this study was to characterize the prevalence of warning symptoms and significant family history in children and young adults who suffered SCA.

\section{Methods}

Data were obtained through a retrospective, crosssectional survey of members of Parent Heart Watch, a national organization of parents and families with a child who suffered SCA. Parent Heart Watch is dedicated to reducing SCA in the young through education, advocacy, and supporting programs targeting prevention. All member families of Parent Heart Watch at the time of this survey $(n=$ 146) were recruited to participate in the study. The comprehensive questionnaire was based on national cardiovascular screening guidelines for young athletes undergoing a preparticipation evaluation..$^{9,11}$ The survey was intended to identify warning symptoms before SCA; family history of cardiovascular disease; the timing of symptoms related to the most recent well-child evaluation, preparticipation evaluation, or physician visit; and the timing of symptom onset relative to SCA. The postmortem evaluation and diagnosis also were investigated.

The survey was distributed using the Catalyst WebQ online survey administration system available through the University of Washington. All member families of Parent Heart Watch were contacted via E-mail and invited to participate in the study. Each E-mail address was assigned a unique survey URL and participant ID number. A recruitment letter was distributed via E-mail and a reminder letter was sent once weekly for 3 weeks to families that did not respond. A paper copy of the survey was provided to participants without access to the Internet.

A similar study has not been performed with the member families of Parent Heart Watch, and the study findings have not been previously reported. This study was approved by the Human Subjects Division of the University of Washington and the Board of Directors of Parent Heart Watch.

\section{Results}

\section{Demographics}

The survey was sent to 146 member families of Parent Heart Watch, with 87 families (60\%) returning a completed survey. Nine cases of SCA were excluded from further analysis, including 5 children who had undergone cardiac surgery, 3 cases of commotio cordis, and one hospital death that was not thought to be caused by SCA. This resulted in 79 cases of SCA in children or young adults from 78 families being included in the analysis.

The average age at the time of SCA was 16 years (range, 5-29 years), 69\% were boys, and 68\% were white (Table 1). Cardiac arrest occurred during or within 1 hour of exercise in $48 \%$ of cases. Only 9 of 79 young persons $(11.4 \%)$ in this study survived their SCA.

\section{Diagnosis}

A broad range of diagnoses were reported by parents as the cause of their child's SCA. The most common diagnosis was autopsy-negative sudden unexplained death $(24 \%)$ followed by hypertrophic cardiomyopathy (23\%), long QT syndrome (11\%), and unknown cause without autopsy (11\%) (Table 2). Of the 61 children and young adults in this study who died of SCA and had an autopsy, a cause of death was identified in only 34 cases (56\%). Eleven of the 27 victims with autopsy-negative sudden unexplained death underwent postmortem genetic testing to evaluate for known genetic causes of SCA. A cause of SCA was identified by postmortem genetic testing in 5 of 11 cases ( $45 \%$ ), including 4 cases of long 
Table 1. Study Population Demographics: 79 Children and Young Adults with Sudden Cardiac Arrest

\begin{tabular}{lc}
\hline Characteristic & \\
\hline Age, years & $7(9)$ \\
$5-10$ & $27(34)$ \\
$11-15$ & $36(46)$ \\
$16-20$ & $6(8)$ \\
$21-25$ & $3(4)$ \\
$26-30$ & \\
Race & $69(87)$ \\
White & $5(6)$ \\
African American & $5(6)$ \\
Hispanic & \\
Sex & $54(68)$ \\
Male & $25(32)$ \\
Female & \\
Relation to exercise & $38(48)$ \\
During or within 1 hour of exercise & $41(52)$ \\
Unrelated to exercise &
\end{tabular}

Values provided as $\mathrm{n}(\%)$.

QT syndrome and one case of arrhythmogenic right ventricular cardiomyopathy (Figure 1). Through subsequent medical evaluation of family members, $15 \%$ of all families reported that another person in the family was diagnosed with the same disorder as their child who suffered SCA.

Table 2. Causes of Sudden Cardiac Arrest in Study Population

Diagnosis

Autopsy negative sudden unexplained death

$19(24)$

Hypertrophic cardiomyopathy

$18(23)$

Long QT syndrome

$8(11)$

Unknown, no autopsy

$9(11)$

Anomalous coronary artery

$4(5)$

Dilated cardiomyopathy

$4(5)$

Arrhythmogenic right ventricular cardiomyopathy

$3(4)$

Myocarditis

3 (4)

Wolff-Parkinson-White syndrome

2 (3)

Mitral valve prolapse

2 (3)

Cardiomyopathy, not otherwise specified

2 (3)

Supraventricular tachycardia

1 (1)

Primary pulmonary hypertension

$1(1)$

Left ventricular hypertrophy

$1(1)$

Coronary artery disease

$1(1)$

Marfan syndrome

Values provided as $\mathrm{n}(\%)$.
Figure 1. Diagnostic evaluation after sudden cardiac arrest.

\section{POST EVENT EVALUATION}

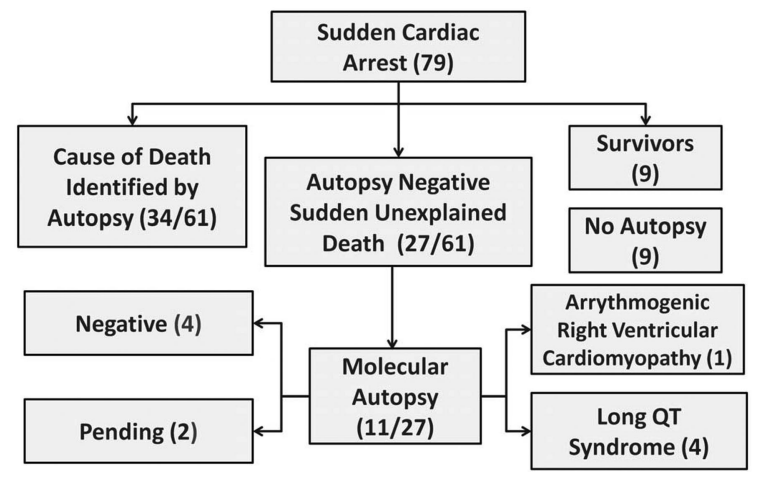

\section{Symptoms}

Parents reported that $72 \%$ of children or young adults represented in this study had at least one cardiovascular symptom before their cardiac arrest. Recent fatigue was the most common symptom reported (44\%). Thirty percent of parents reported near-syncope/lightheadedness in their child before SCA, $28 \%$ of parents reported their child complained of chest pain or palpitations, and $23 \%$ reported their child complained of shortness of breath. Thirteen percent of parents reported at least one episode of unexplained seizure activity and $18 \%$ reported an episode of syncope before SCA. Parents reported the initial onset of cardiovascular symptoms occurred on average 30 months (range, 19 to 71 months) before their child's SCA (Table 3).

A total of $24 \%$ of the SCA victims had at least one objective warning event, either syncope or unexplained seizure activity, that remained undiagnosed as a cardiac disorder before SCA. On average, these children had 2.6 episodes (range, 1 to 10 episodes) of syncope or unexplained seizure activity before their final SCA. Syncope was reported to occur first an average of 4 years before SCA, and unexplained seizure activity first occurred approximately 6 years before SCA (Table 3).

A preparticipation evaluation was completed in $61 \%$ of children an average of 15.6 months before SCA, and a well-child evaluation was completed in $60 \%$ of children an average of 12.6 months before SCA. Fifty-one percent of children were reported to have at least one cardiovascular symptom before their last physician visit (Table 3), and $41 \%$ of 
Table 3. Reported Symptom Prevalence in Children and Young Adults with Sudden Cardiac Arrest (SCA)*

\begin{tabular}{lcccc}
\hline Symptom & $\begin{array}{c}\text { Study Population with } \\
\text { Symptom (\%) }\end{array}$ & $\begin{array}{c}\text { SCA Victims with Symptoms } \\
\text { before Their Most Recent } \\
\text { Physician Visit (\%) }\end{array}$ & $\begin{array}{c}\text { Events, n } \\
\text { (range) }\end{array}$ & $\begin{array}{c}\text { Symptom Onset before } \\
\text { SCA (months) }\end{array}$ \\
\hline Fatigue & 44 & 25 & NA & 21 \\
Near-syncope/lightheadedness & 30 & 22 & NA & 30 \\
Chest pain/discomfort & 28 & 20 & NA & 26 \\
Palpitations & 28 & 17 & NA & 25 \\
Heart murmur & 24 & - & NA & 89 \\
Shortness of breath & 23 & 20 & NA & 19 \\
Tire more easily than friends & 22 & - & NA & NA \\
Syncope & 18 & 11 & $2.9(1-5)$ & 47 \\
Unexplained seizure activity & 13 & - & NA & 71 \\
Decrease in physical activity & 11 & - & NA & NA \\
Hypertension & 3 & 51 & - & 25 \\
One of the above symptoms & 72 & & -
\end{tabular}

*Timing of symptoms related to most recent physician visit and SCA is provided.

NA, not applicable.

families reported that at least one symptom was brought to the attention of their physician.

\section{Family History}

Forty percent of the study population identified at least one significant family history component that was present before their child's SCA. Twentyseven percent of the study population reported a family member who died of a heart condition before the age of 50 , of whom $15 \%$ had a cardiac pacemaker, $14 \%$ had unexplained fainting, and 5\% had an unexplained seizure diagnosis. A small number of families knew of a specific cardiac condition in their family before their child's SCA (Table 4).

\section{Discussion}

The prevalence of warning signs and symptoms in children and young adults with occult cardiovascular disease is poorly understood. As demonstrated in this study, an important proportion of children and young adults with cardiovascular disorders that go on to experience SCA may have warning symptoms or a significant family history of premature cardiovascular disease that could be identified through appropriate screening.

Routine well-child visits are universally conducted in the United States and focus on a continuum of disease detection, disease prevention, health promotion, and anticipatory guidance throughout childhood and adolescence. ${ }^{15}$ Nevertheless, current standards in medical education lack any substantial emphasis in detecting cardiovascular warning symptoms in children, and the recommended cardiac evaluation in children is often limited to the presence of cyanosis, chest pain, asymmetric pulses, or a detectable murmur. ${ }^{15}$

A greater emphasis has been placed on the cardiovascular evaluation of children and young adults participating in competitive athletics. The American College of Cardiology states that the primary objective of the preparticipation evaluation of ath-

\section{Table 4. Reported Family History of Children and Young Adults with Sudden Cardiac Arrest}

\begin{tabular}{lc}
\hline Family History & \\
\hline Died of cardiac disease before age 50 years & $21(27)$ \\
Coronary artery disease & $18(23)$ \\
Mitral valve prolapse & $12(15)$ \\
Cardiac pacemaker & $12(15)$ \\
Unexplained fainting & $11(14)$ \\
Unexplained seizure diagnosis & $4(5)$ \\
Drowning or near drowning event & $4(5)$ \\
Wolff-Parkinson-White syndrome & $3(4)$ \\
Implanted Cardiac defibrillator & $1(<1)$ \\
Hypertrophic cardiomyopathy & $1(<1)$ \\
Long QT syndrome & $1(<1)$ \\
Dilated cardiomyopathy & $1(<1)$ \\
Anomalous coronary artery & $1(<1)$ \\
Aortic rupture/Marfan's syndrome & $1(<1)$ \\
At least one of the above family history & $32(40)$ \\
components & \\
\hline
\end{tabular}

Values provided as $\mathrm{n}(\%)$. 
letes is the detection of silent cardiovascular abnormalities that predispose to SCD. ${ }^{16}$ In 1996 the AHA provided consensus recommendations for the preparticipation cardiovascular evaluation of athletes, which includes 12 elements of a personal history, family history, and the physical examination. ${ }^{9,17}$ These consensus recommendations were reaffirmed in 2007. Unfortunately, more than a decade later, use of a comprehensive history questionnaire in the cardiovascular screening of athletes has not been adopted widely into practice by primary care providers. In the United States, 35\% of states allow nonphysician examiners with little cardiovascular training to perform a preparticipation evaluation, and only $81 \%$ of states have adequate questionnaires based on the AHA recommendations. $^{12}$

Despite longstanding recommendations for a history- and physical-based preparticipation evaluation, there are no outcomes-based studies that demonstrate that the traditional preparticipation evaluation is effective in preventing or detecting athletes at risk for sudden death. A 1996 US study reviewed sudden deaths in 158 trained athletes, 134 of whom were though to have suffered SCA of a cardiovascular cause. ${ }^{4}$ Only $3 \%$ of athletes who suffered SCA were suspected of having cardiovascular disease, and only one athlete with a specific cardiac anomaly was correctly identified by a preparticipation screen. Although the details and adequacy of the preparticipation evaluations performed were not reported, the authors summarized that preparticipation screening seemed to be of limited value for the identification of underlying cardiovascular abnormalities. ${ }^{4}$

A significant challenge to screening is that asymptomatic, apparently healthy children and young adults may harbor unsuspected cardiovascular disease. ${ }^{18,19}$ Sudden death is the first manifestation of a cardiac disorder in $50 \%$ to $80 \%$ of young athletes. ${ }^{4-7}$ Only $21 \%$ of athletes who died from hypertrophic cardiomyopathy ${ }^{4}$ and $44 \%$ of athletes who died of an anomalous coronary artery ${ }^{6}$ had any signs or symptoms of cardiovascular disease in the years before their death. Sudden death also was found to be the sentinel cardiovascular event in $80 \%$ to $90 \%$ of autopsy-negative sudden unexplained death. ${ }^{7,20}$

However, symptoms in children and young adults with primary structural or electrical cardiac abnormalities may, in fact, be relatively common but be misinterpreted or disregarded by medical providers. A 1996 report summarized 9 publications detailing 469 sudden deaths from cardiac causes in young persons. ${ }^{21}$ These studies collectively reported preceding symptoms of dizziness, chest pain, syncope, palpitations, dyspnea, and a family history of sudden death from a cardiac cause in $25 \%$ to $61 \%$ in their study populations. ${ }^{21}$ Wisten and Messner ${ }^{18}$ investigated symptoms in a Swedish cohort of 15 to 35 year olds who had suffered SCD and reported that 92 of 162 individuals with SCD (57\%) had preceding symptoms that often were misinterpreted. In this study, $72 \%$ of children were reported to have at least one cardiovascular symptom before SCA. Even less information is known about the prevalence of a family history of cardiovascular disease in children that go on to experience SCA. In this study, $40 \%$ of the study population reported at least one significant family history component present before their child's SCA.

The relative infrequency of SCA in children and young adults makes prospective study of warning symptoms very challenging. This study attempted to examine a large cohort of families affected by SCA. However, the limitations of this study should be recognized in the interpretation of the results. A survey of families and parents who have lost a child to SCA has a high potential for recall and reporting bias when inquiring about the presence of warning symptoms before SCA. Thus, the reported presence of warning symptoms before SCA in this study may be overestimated. The reported duration of time between initial symptom onset and SCA also may be overestimated. This study was not designed to compare differences among the etiologies reported, and it is possible that different causes of SCA may affect the likelihood of symptoms. There was no control group without SCA for the comparison of symptom and family history frequency, and the study was not designed to determine the sensitivity, specificity, or positive and negative predictive values of each symptom or family history element. It is unknown if asking a general population of children and young adults questions about subjective cardiovascular symptoms such as chest pain and lightheadedness will yield an acceptable accuracy to identify children at elevated risk for SCA or if these symptoms are just too common in the pediatric population to make it a valuable screening question. 
However, the number of victims with multiple episodes of an objective warning event such as syncope or unexplained seizure activity that went undetected as cardiovascular disease before their death is alarming. Syncope and unexplained seizure activity represent distinct events in a person's medical history and are therefore less likely to be affected by recall bias. The reporting of a family history of cardiovascular disease is also less likely to be inaccurate, although many of the families may have learned of their family history of cardiovascular disease after their child's event. Based on the results of this study, if physicians at well-child and preparticipation visits emphasize only 3 objective historical features (history of syncope, history of unexplained seizure activity, and a family history of sudden death caused by heart disease at an age younger than 50 years), approximately one third of children and young adults with cardiovascular disorders at elevated risk for SCA may be detected, assuming an appropriate diagnostic evaluation then is conducted.

Syncope in the young usually represents neurocardiogenic syncope, also known as vasovagal syncope, and often is triggered by specific events such as fear, pain, excitement, blood draws, or prolonged standing. ${ }^{22}$ Premonitory symptoms before collapse are common in vasovagal syncope and include lightheadedness, dizziness, diaphoresis, nausea, and tunnel vision. In contrast, individuals with syncope from a cardiac disorder at risk for SCA usually have an abrupt collapse without warning because of the onset of a potentially lethal ventricular arrhythmia.

The diagnosis of vasovagal syncope is made by a clinical history consistent with neurocardiogenic syncope, a normal physical examination, and a normal 12-lead electrocardiogram (ECG) to exclude findings suggestive of underlying cardiac disorders, such as ion channel disorders or cardiomyopathies. Routine testing with ECG is recommended during the initial evaluation of any child or young adult with syncope. Ritter et $\mathrm{al}^{23}$ reviewed the records of 480 pediatric patients (median age, 13 years) who presented with syncope. A cardiac etiology for syncope was identified in 22 patients (5\%), including long QT syndrome $(\mathrm{n}=14)$, arrhythmias $(\mathrm{n}=6)$, and cardiomyopathies $(\mathrm{n}=2)$. An abnormal history (exercise-induced syncope or family history of sudden death), physical examination, or ECG identified 21 of the 22 patients with a cardiac cause of syncope (96\% sensitivity; 99\% negative predictive value) ${ }^{23}$ If the history and physical examination are typical for neurocardiogenic syncope and the ECG is normal, further testing generally is not needed.

Syncope occurring during exercise is an ominous sign and warrants a high index of suspicion for underlying cardiac disease. In a review of 474 athletes with a history of syncope or near-syncope found during preparticipation screening, $33 \%$ with syncope that occurred during exercise were found to have a structural cardiac disease known to cause SCD. ${ }^{24}$ The diagnostic workup of exertional syncope usually is performed in consultation with a cardiologist and should include an ECG, echocardiogram, stress ECG, and possibly advanced cardiac imaging (such as cardiac magnetic resonance imaging or computed tomography) to rule out rare structural abnormalities such as arrhythmogenic right ventricular cardiomyopathy or congenital coronary artery anomalies.

Seizures can be difficult to distinguish from syncope and thus should be part of the differential diagnosis. It should be noted that myoclonic movements caused by cerebral hypoperfusion can mimic true seizures. The clinical difference between the 2 is that if cerebral hypoperfusion is causing the myoclonic movements, the individual usually collapses first and then develops involuntary body movements seconds later, whereas with a true seizure the myoclonic movements would begin concurrently with the loss of postural tone and consciousness. ${ }^{25}$ SCA is commonly mistaken for a seizure. ${ }^{26}$ Brief seizure-like activity or involuntary myoclonic jerks are reported in approximately $50 \%$ of young athletes with SCA. ${ }^{27,28}$ Any patient with unexplained seizure activity should have a cardiovascular workup, including a 12-lead ECG and an echocardiogram at a minimum.

Postmortem genetic screening is also important in the identification of the cause of SCA and of other at-risk family members with the same cardiac condition. In studies performing postmortem genetic testing (so-called "molecular autopsy") in cases of autopsy-negative sudden unexplained death, more than one third of cases were found to have a pathogenic cardiac ion channel mutation. ${ }^{20,29}$ Similarly, in this study, a cause of SCA was identified by postmortem genetic testing in $45 \%$ of cases with autopsy-negative sudden unexplained death. A definite diagnosis of SCA based on genetic testing allows for possible treatment of and improved risk reduction in family members. Further research is needed to bet- 
ter understand the prevalence of potential cardiovascular warning symptoms in a general pediatric population and to determine the questions with the highest likelihood of identifying individuals with underlying cardiac conditions that predispose to sudden death.

\section{Conclusions}

Many children and young adults who suffer SCA are reported to have cardiac symptoms or a family history of cardiac disease that could be detectable through screening. Syncope and unexplained seizure activity are objective events but often go unrecognized as ominous signs of underlying cardiovascular disease. Utilization of a comprehensive questionnaire to conduct cardiovascular risk assessments in children during well-child and preparticipation evaluations may improve detection of children at elevated risk and help prevent sudden death. Improved education of primary care and sports physicians conducting these evaluations regarding the warning symptoms, family history, and secondary investigations that may indicate the presence of a lethal cardiovascular abnormality is needed. In addition, education of the public, parents, and children regarding SCA in the young and the association of specific symptoms or a positive family history of early SCA may help identify those at risk and prevent tragedies. Research to determine the sensitivity and specificity of screening with personal and family history questions also is needed and may shape future recommendations.

The authors would like to thank Parent Heart Watch for their participation in this study and their continued efforts in the prevention of sudden cardiac death in the young.

\section{References}

1. Maron BJ, Doerer JJ, Haas TS, Tierney DM, Mueller FO. Sudden deaths in young competitive athletes: analysis of 1866 deaths in the United States, 1980-2006. Circulation 2009;119:1085-92.

2. Heron M, Hoyert DL, Murphy SL, Xu J, Kochanek KD, Tejada-Vera B. Deaths: final data for 2006. Natl Vital Stat Rep 2009;57:1-134.

3. Campbell RM, Berger S, Drezner J. Sudden cardiac arrest in children and young athletes: the importance of a detailed personal and family history in the pre-participation evaluation. Br J Sports Med 2009;43:336-41.

4. Maron BJ, Shirani J, Poliac LC, et al. Sudden death in young competitive athletes. Clinical, demographic, and pathological profiles. JAMA 1996;276:199-204.
5. Corrado D, Basso C, Fontaine G. Clinical profile of young competitive athletes who died suddenly of arrhythmogenic right ventricular cardiomyopathy/ dysplasia: a multicenter study. Pacing Clin Electrophysiol 2002;25:544.

6. Basso C, Maron BJ, Corrado D, et al. Clinical profile of congenital coronary artery anomalies with origin from the wrong aortic sinus leading to sudden death in young competitive athletes. J Am Coll Cardiol 2000;35:1493-501.

7. Eckart RE, Scoville SL, Campbell CL, et al. Sudden death in young adults: a 25-year review of autopsies in military recruits. Ann Intern Med 2004;141:829-34.

8. Ljungqvist A, Jenoure P, Engebretsen L, et al. The International Olympic Committee (IOC) consensus statement on periodic health evaluation of elite athletes. Br J Sports Med 2009;43:631-43.

9. Maron BJ, Thompson PD, Ackerman MJ, et al. Recommendations and considerations related to preparticipation screening for cardiovascular abnormalities in competitive athletes: 2007 update: a scientific statement from the American Heart Association Council on Nutrition, Physical Activity, and Metabolism: endorsed by the American College of Cardiology Foundation. Circulation 2007;115:1643-55.

10. Corrado D, Pelliccia A, Bjornstad HH, et al. Cardiovascular pre-participation screening of young competitive athletes for prevention of sudden death: proposal for a common European protocol. Consensus statement of the Study Group of Sport Cardiology of the Working Group of Cardiac Rehabilitation and Exercise Physiology and the Working Group of Myocardial and Pericardial Diseases of the European Society of Cardiology. Eur Heart J 2005;26:516-24.

11. American Academy of Family Physicians, American Academy of Pediatrics, American College of Sports Medicine, American Medical Society for Sports Medicine, American Orthopaedic Society for Sports Medicine, American Osteopathic Association of Sports Medicine. Preparticipation physical evaluation. 3rd ed. New York: McGraw-Hill; 2005.

12. Glover DW, Glover DW, Maron BJ. Evolution in the process of screening United States high school student-athletes for cardiovascular disease. Am J Cardiol 2007;100:1709-12.

13. Glover DW, Maron BJ. Profile of preparticipation cardiovascular screening for high school athletes. JAMA 1998;279:1817-9.

14. Gomez JE, Lantry BR, Saathoff KN. Current use of adequate preparticipation history forms for heart disease screening of high school athletes. Arch Pediatr Adolesc Med 1999;153:723-6.

15. Part XIX: the cardiovascular system. In: Kliegman R, Behrman RE, Jenson HB, Stanton BMD, eds. Nelson Textbook of Pediatrics. 18th ed. Philadelphia: Saunders; 2007.

16. Maron BJ, Douglas PS, Graham TP, et al. Task Force 1: preparticipation screening and diagnosis of 
cardiovascular disease in athletes. J Am Coll Cardiol 2005;45:1322-6.

17. Maron BJ, Thompson PD, Puffer JC, et al. Cardiovascular preparticipation screening of competitive athletes. A statement for health professionals from the Sudden Death Committee (clinical cardiology) and Congenital Cardiac Defects Committee (cardiovascular disease in the young), American Heart Association. Circulation 1996;94:850-6.

18. Wisten A, Messner T. Symptoms preceding sudden cardiac death in the young are common but often misinterpreted. Scand Cardiovasc J 2005;39:143-9.

19. Wilson MG, Basavarajaiah S, Whyte GP, et al. Efficacy of personal symptom and family history questionnaires when screening for inherited cardiac pathologies: the role of electrocardiography. $\mathrm{Br} \mathrm{J}$ Sports Med 2008;42:207-11.

20. Tester DJ, Spoon DB, Valdivia HH, et al. Targeted mutational analysis of the RyR2-encoded cardiac ryanodine receptor in sudden unexplained death: a molecular autopsy of 49 medical examiner/coroner's cases. Mayo Clin Proc 2004;79:1380-4.

21. Liberthson RR. Sudden death from cardiac causes in children and young adults. N Engl J Med 1996;334: $1039-44$.
22. Grubb BP. Clinical practice. Neurocardiogenic syncope. N Engl J Med 2005;352:1004-10.

23. Ritter S, Tani LY, Etheridge SP, et al. What is the yield of screening echocardiography in pediatric syncope? Pediatrics 2000;105:E58.

24. Colivicchi F, Ammirati F, Santini M. Epidemiology and prognostic implications of syncope in young competing athletes. Eur Heart J 2004;25:1749-53.

25. Link MS, Estes NA 3rd. How to manage athletes with syncope. Cardiol Clin 2007;25:457-66.

26. Hauff SR, Rea TD, Culley LL, et al. Factors impeding dispatcher-assisted telephone cardiopulmonary resuscitation. Ann Emerg Med 2003;42:731-7.

27. Drezner JA, Rao AL, Heistand J, et al. Effectiveness of emergency response planning for sudden cardiac arrest in united states high schools with automated external defibrillators. Circulation 2009;120:518-25.

28. Drezner JA, Rogers KJ. Sudden cardiac arrest in intercollegiate athletes: detailed analysis and outcomes of resuscitation in nine cases. Heart Rhythm 2006;3:755-9.

29. Tester DJ, Ackerman MJ. The role of molecular autopsy in unexplained sudden cardiac death. Curr Opin Cardiol 2006;21:166-72. 